\title{
A balanced approach to race in the treatment of personality disorder
}

\author{
Piyal Sen \& Dave Ramaswamy
}

\begin{abstract}
SUMMARY
This case illustrates a clinical management problem in which a terminally ill psychiatric patient racially abuses the Black staff caring for him. The clinical team has to tread a fine line between supporting staff victimised by the patient and ensuring his quality of life and care. It is important that patients' (and staff's) sensitivities relating to race and ethnicity are openly discussed at an early stage, so that appropriate clinical management can be negotiated.
\end{abstract}

\section{DECLARATION OF INTEREST}

P. S. was the patient's consultant at the time and D. R. was the ward manager. Both authors have subsequently moved on to other jobs.

Race and mental health is a highly contentious political topic (Singh 2006). Many concerns centre on allegations of institutional racism within psychiatry, experienced by Black patients in a predominantly White psychiatric establishment. This article describes a reverse scenario, that of a White patient's racism towards a predominantly Black staff group. The patient discussed here died while in hospital and his details have been altered to ensure that he cannot be recognised.

\section{Background}

The patient was detained in a medium secure forensic unit specialising in the treatment of personality disorder. He had previously been in high security, admitted on a hospital order with restrictions under Sections 37 and 41 of the Mental Health Act 1983 following a conviction for manslaughter. He had spent virtually his entire adult life in custody and had a diagnosis of dissocial (antisocial) personality disorder (ICD10, F60.2; World Health Organization 1992). He also suffered from depression and had been treated with antidepressants, mood stabilisers and electroconvulsive therapy. He had a long history of substance misuse.

When he moved to medium security, he was in his early 40s, after spending more than 20 years in maximum security. The severe emphysema he had had before the transfer worsened and he required a number of hospital admissions, including a period in critical care. This deterioration meant that he found it increasingly difficult to walk more than a few steps before he started to feel breathless. His frustration grew and he became more and more verbally abusive towards staff and fellow patients. The combination of his physical and psychological needs necessitated almost one-to-one nursing.

The ratio of Black to White staff on the ward was about $4: 1$. This meant that the patient was often nursed by Black staff on an individual basis. He consistently resorted to racist abuse of these individuals. When he was admitted to hospital for treatment of periodic chest infections, he would demand to have only White staff at his bedside. He expressed fears of dying in front of staff from Black and minority ethnic groups.

The hospital had a zero-tolerance policy towards racism. Any racist incident had to have consequences for the perpetrator, which could include reporting the matter to the police. However, because of the clinical condition of the patient, he did not have any privileges (such as leave) that could be withdrawn. A police prosecution carried little weight for him, as his legal status was unlikely to change. He was very open when challenged about his racism, saying that he did it deliberately so that Black staff were not willing to work with him.

\section{The clinical issue}

The dilemma for the clinical team was a clinical as well as an ethical one. The patient was clearly deteriorating physically and there was a case for him to be cared for by staff with whom he felt most comfortable, on quality of life grounds.

However, it was not quite as simple as that. The patient was in a long-term care setting. All the other patients on that ward, which was a specialist personality disorder ward, were also of White ethnicity, and quite a few of them did not like to have Black staff care for them. The clinical team would have to consider how to respond if similar demands were made by other patients.

There was also the matter of staff morale. Most of the staff felt that the clinical team was only perpetuating his racist beliefs by 'giving in' to his demands, and this was not felt to be a helpful way
Piyal Sen is a consultant forensic psychiatrist who runs a personality disorder service in a low-security setting at St Andrew's Healthcare, Clare House, Essex. Dave Ramaswamy is a senior nurse (operations) at Cheswold Park Hospital, Doncaster. Correspondence Dr Piyal Sen, Department of Forensic Psychiatry, St Andrew's Healthcare, Clare House, Pound Lane, North Benfleet, Essex SS12 9JP, UK. Email: psen@ standrew.co.uk 
MCQ answers

$1 \mathrm{c} \quad 2 \mathrm{~d} \quad 3$ e $\quad 4$ e to support staff. The clinical team thus had to find a way that would offer the best balance between the rights of this patient, of the other patients and of the nursing staff.

\section{Discussion}

The term 'ecosystem distrust' has been used to describe a self-protective defensiveness, a syndrome characterised by lack of trust in other people, suspicion of their motives, a sense of individual powerlessness and a sense that caution is necessary to avoid trouble (Triandis 1976). This is normally used to describe the experience of Black people in an oppressive White environment. It is possible that our patient was beginning to feel some of this in an environment dominated by Black staff, especially as he felt increasingly vulnerable because of his deteriorating physical health. Ethnicity and culture strongly influence human behaviour and a proper understanding of the influence of culture on paranoia, even if it is non-pathological, should form a mandatory part of any psychiatric assessment in a multicultural setting (Sen 2006). Thus, the cultural context of the patient's mistrust of services cannot be ignored.

From the nursing perspective, racism against nurses is a persistent problem in the healthcare services (Larsen 2006). In the UK, there has been frustration at government's continued failure to legislate against racial abuse, and reputable organisations such as the Royal College of Nursing have called for an action plan to support Black staff (Stone 2007; Kendall-Raynor 2008; Sprinks 2008). Scenarios in which a patient objects to being cared for on the basis of a staff member's race are not at all uncommon (Dimond 2006). Thus, it was absolutely essential for the clinical team to be supportive of the nursing team, who had been persistently racially abused by this patient.

\section{Clinical approach}

Initially, the clinical team set up a protocol with the patient by which he would deal directly with the ward manager regarding any concerns about any member of the nursing team. However, only clinical and professional matters could be raised. Issues such as race could not be introduced into these discussions. He was also encouraged to bring up any general ward-related issues in the community meetings attended by members of the multidisciplinary team and the patients.

Unfortunately, this approach did not work as the concerns the patient took to the ward manager continued to be race-related and he did not attend the community meetings. The clinical team then discussed the matter in a team meeting in which all views and options were thoroughly aired and discussed. It was noted that, although the patient always preferred White staff, he had a tolerable relationship with some of the Black members of the nursing team. It was agreed that a list could be drawn up of all the staff members (both Black and White) with whom he had a civil relationship. A general protocol was drawn up that the staff members on this list would provide most of his care, particularly when he went into the general hospital. This meant that his care was not being provided exclusively by White staff, which would send out the wrong message, but by staff who would best be able to fulfil his clinical needs because of their therapeutic relationship with him.

The consultant and the ward manager explained the rationale for this protocol in detail to the nursing team. The matter was also discussed in supervision and support sessions with junior staff who had expressed reservations about the patient's care. Once the whole clinical team was in agreement, the patient was approached. It was clearly explained to him that this was the fairest arrangement, as even White staff were not willing to work with him any more because of his overdependence on them and what they perceived to be time taken away from other patients because of this. He saw in it his only chance of not dying in front of Black staff and accepted the arrangement.

The patient eventually died relatively peacefully following one of numerous admissions to critical care after he had developed a chest infection. His parents were with him at the time and were very appreciative of the care he had received from the multicultural staff team. They mentioned this at his funeral, which was attended by a number of members of the clinical team as well as fellow patients.

\section{Concluding remarks}

What worked well in this case was a balanced multidisciplinary approach to a sensitive clinical issue, ultimately leading to a satisfactory outcome. Nevertheless, for a while the patient had managed to split the clinical team, particularly the nursing team, on the highly contentious issue of race. He was able successfully to exploit staff sensitivity on this matter, an area in which patients with personality disorder are often very skilled.

In such cases, it is very important to raise uncomfortable clinical issues such as race and culture at an early stage and to explore staff and patient sensitivities about it. The dilemmas involved in offering the best clinical care to the patient while ensuring that staff feel adequately supported should be openly discussed. It then 
becomes easier to arrive at the clinical option offering the best balance between the apparently competing demands of staff and patients. As the psychiatric system in the UK gears up to treat more patients with personality disorder in multicultural clinical settings, this clinical scenario will become increasingly common.

\section{References}

Dimond B (2006) A patient objects to being cared for by you on the basis of your race: what should you do? Nursing Times 102: 10.

Kendall-Raynor P (2008) Action plan to support black staff as research shows racism persists. Nursing Standard 22: 6 .
Larsen J (2006) Overseas nurses: tackling discrimination. Nursing and Residential Care 8: 368-70.

Sen P, Chowdhury AN (2006) Culture, ethnicity and paranoia. Current Psychiatry Reports 8: 174-8.

Singh SP, Burns T (2006) Race and mental health: is there more to race than racism. BMJ 333: 648-51.

Sprinks J (2008) Diversity champions needed to tackle racism against NHS staff. Nursing Standard 23 (4): 12-3.

Stone $\mathrm{R}$ (2007) The government's continued failure to legislate against racial abuse. Mental Health Today 41: 1474

Triandis H (1976) Variations in Black and White Perceptions of the Social Environment. University of Illinois Press.

World Health Organization (1992) The ICD-10 Classification of Mental and Behavioural Disorders. World Health Organization.

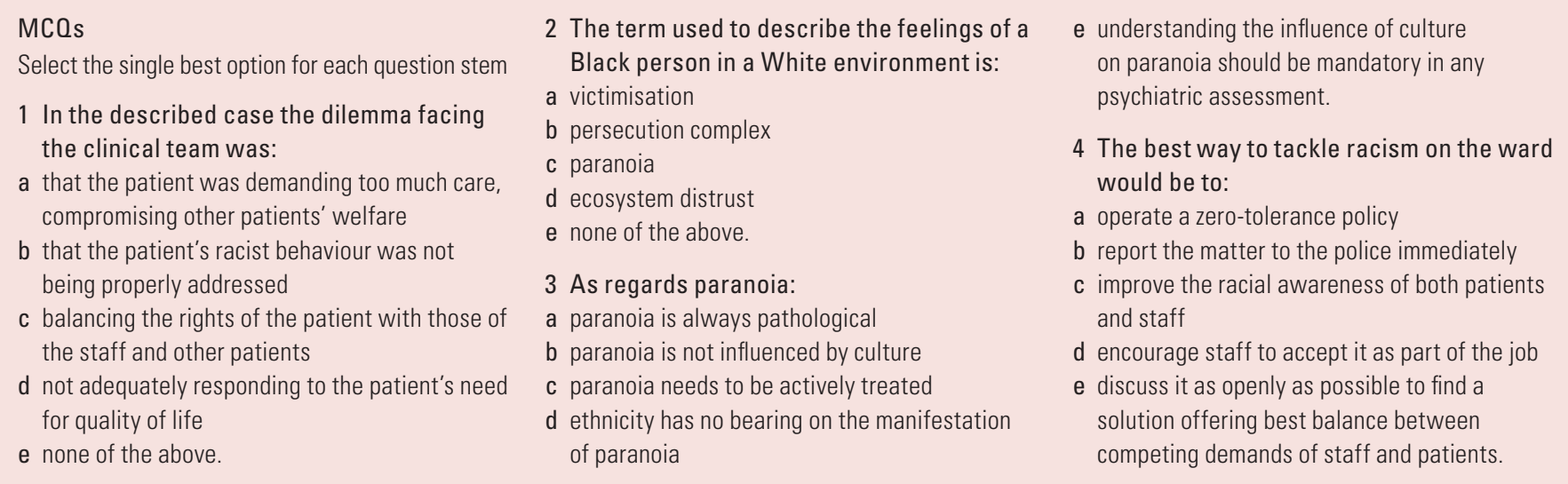

